\title{
Potential Correlations between Unexplained Experimental Observables and Hot Projectile-Like Fragments in Primary Interactions above $E_{\mathrm{CM}} / \mathrm{u} \approx 150 \mathrm{MeV}$
}

\author{
Eberhard Ganssauge ${ }^{1}$, Wolfram Westmeier ${ }^{2,3^{*}}$, Reinhard Brandt ${ }^{2}$ \\ ${ }^{1}$ Fachbereich Physik, Philipps University, Marburg, Germany \\ ${ }^{2}$ Fachbereich Chemie, Philipps-University, Marburg, Germany \\ ${ }^{3}$ Dr. Westmeier GmbH, Ebsdorfergrund, Germany \\ Email: *westmeier@t-online.de
}

Received July 22, 2013; revised August 25, 2013; accepted September 11, 2013

Copyright (C) 2013 Eberhard Ganssauge et al. This is an open access article distributed under the Creative Commons Attribution License, which permits unrestricted use, distribution, and reproduction in any medium, provided the original work is properly cited.

\begin{abstract}
An enhanced neutron production and an enhanced nuclear destruction due to secondary fragments have been observed in very thick targets irradiated with high energy ions. This enhancement is beyond theoretical calculations and it is an unresolved problem. It is observed only when primary ion interactions exceed an energy threshold $\left(E_{\mathrm{CM}} / u \approx 150 \mathrm{MeV}\right)$. Investigations using nuclear emulsions for very high-energy nuclear reactions suggest that two distinctly different classes of relativistic projectile-like fragments are emitted in primary interactions: a "cool" channel with a temperature of $\left(T(p)_{\text {cool }} \approx 10 \mathrm{MeV}\right)$, and a "hot" channel with $\left(T(p)_{\text {hot }} \approx 40 \mathrm{MeV}\right.$. This second reaction class may induce the above mentioned enhanced reactions of secondary fragments, thus being responsible for unresolved problems. This assumption should be studied in further experiments. Nuclear interactions of secondary particles in thick targets are of interest, in particular in view of radiation protection needs for high energy and high intensity heavy ion accelerators. Many basic ideas of this paper go back to the late Professor E. Schopper (Frankfurt).
\end{abstract}

Keywords: Very High Energy Projectile; Hot Projectile Fragments; Cool Target Fragments; Unresolved Problems

\section{Introduction}

Since around 1950, researchers have been unable to resolve problems in explaining observed phenomena in nuclear reactions at high energies in very thick targets.

In a recent publication on "Correlations in nuclear interactions between $E_{\mathrm{CM}} / u$ and unexplained experimental observables" [1] it is pointed out that these observations are restricted to high energy interactions induced by primary ions in medium and heavy element target nuclei above a threshold of $E_{\mathrm{CM}} / u \approx 150 \mathrm{MeV}$ for the primary nuclear interaction. Enhanced neutron emission and enhanced nuclear destruction of target nuclei in secondary reactions are observed which significantly exceed model calculation. The concept of "limiting fragmentation" in spallation reactions does not hold in these thick target interactions any longer. In a preceding publication the enhanced "Neutron production in thick targets irradiated with high energy ions" [2] and radiochemical spallation yields were analyzed using advanced computer simula-

"Corresponding author. tions such as MCNPX 2.7a. The authors concluded that enhanced neutron production (quote) "may have an impact on radiation safety protection issues for future heavy ion accelerators" under construction. A systematic review on "Interactions of relativistic heavy ions in thick heavy element targets and some unresolved problems" [3] has been published earlier.

In 1996, the late Professor E. Schopper (Frankfurt) informed the Marburg group in a private letter that Baumgardt, Friedlander and Schopper had shown in 1984 [4] that two distinctly different exit channels had been observed in the reaction of $100 \mathrm{GeV}{ }^{56} \mathrm{Fe}$ ions with nuclear emulsion. Fifteen years later, we are following up on Prof. Schopper's suggestion to continue our work in this direction. One channel was "cold" and consistent with wellknown spallation reactions. The other channel was "hot" and not compatible with any model calculation. This observation was confirmed by several experimentalists. However, it was in the following completely ignored by all scientists, even by Friedlander himself. We will re-investigate the phenomena observed in [4]. Our interest is 
focused on the FIRST nuclear interaction of $100 \mathrm{GeV}$ ${ }^{56} \mathrm{Fe}$ observed in nuclear emulsions, following a suggestion of the late Professor Schopper:

"Is this 'hot' channel in the $1^{\text {st }}$ interaction responsible for the observation of unresolved problems due to interactions of secondary fragments?"

Projectile fragments emitted in the "hot" exit channel of the first interaction will induce secondary interactions in thick targets which may be the source of unresolved problems. The situation will be described, but no definite solution can be given yet.

\section{Primary Interactions Produce "Cool" and "Hot" Relativistic Projectile-Like Fragments}

Detailed investigations with nuclear emulsions of primary interactions where $E_{\mathrm{CM}} / u$ exceeds $150 \mathrm{MeV}$ are available only for irradiations with either $100 \mathrm{GeV}{ }^{56} \mathrm{Fe}$ or $72 \mathrm{GeV}^{40} \mathrm{Ar}$ projectiles. These reactions were studied about three decades ago [4-10]. For all relevant interactions with target atoms in emulsion $\left({ }^{107,109} \mathrm{Ag},{ }^{79,81} \mathrm{Br},{ }^{16} \mathrm{O}\right.$, ${ }^{14} \mathrm{~N},{ }^{12} \mathrm{C}$ ), but not with protons, the energy threshold of $E_{\mathrm{CM}} / u \approx 150 \mathrm{MeV}$ is clearly exceeded.

Baumgardt, Friedlander, and Schopper [4] showed that all heavy target elements in nuclear emulsion with a mass $A \geq 12$ amu are involved in primary interactions. However, they studied only those nuclear interactions, (observed under an optical microscope as a "star" in a nuclear emulsion) where target-like products, visible as a number of heavy tracks, either black or grey, could be separated experimentally in a clear manner from relativistic projectile-like fragments moving forward as minimumionizing ions (visible as thin tracks). An example of such an event is shown in Figure 1. The authors of [4] restricted their analysis further to such events having at least one projectile-like fragment with $Z \geq 3$ and at least one projectile-like helium-ion $(Z=2)$. They measured the pseudo-rapidity (related to the transverse-momentum $P_{t}$ ) only for these helium-ions and treated the angular distribution of the He projectile fragments within the framework of thermodynamic models. The results are presented in convenient parameter space like squared transverse momenta per nucleon using a quantity $Q$, defined as

$$
Q=\left(P_{t} / A\right)^{2}
$$

where $A=4$ is the mass of the outgoing particle.

In their analysis the transverse momentum, $P_{t}$, for each relativistic $\alpha$-projectile fragment was determined by the measurement of its polar angle $\Theta$ with respect to the beam direction. Assuming beam velocity for the He fragments they introduced as a good approximation

$$
Q \approx q=M_{p}^{2} \cdot\left(\gamma^{2}-1\right) \cdot \sin ^{2} \Theta
$$

with $\gamma$ being the Lorentz factor of the projectile, $M_{p}$ is the

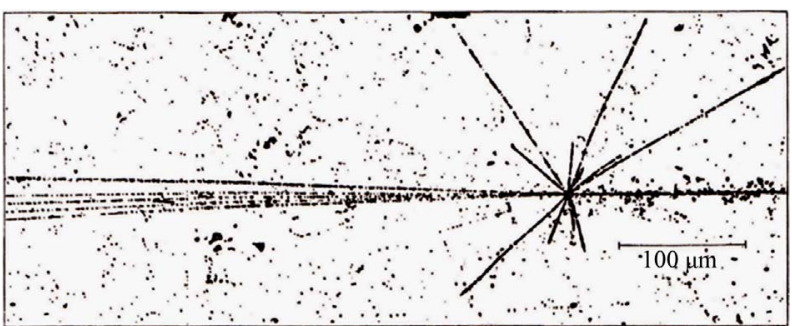

Figure 1. An example of an Ar-induced interaction "star" (or event) in Ilford G5 nuclear emulsion. The relativistic Ar-ion enters from the right and moves to the left. Clearly seen are two different types of fragments. The target-like products are emitted more or less isotropically from a point source as black or grey prongs. The projectile-like fragments are emitted as minimum-ionizing particles leaving tracks into a small forward cone [5].

proton mass and $\Theta$ is fragment's polar angle. In nomenclature and analysis the authors of [4] follow a longstanding tradition of scientists in the field of nuclear emulsion studies. The interested reader may consult the textbook of Powell et al. [6]. If the emission of the $\mathrm{He}$ fragments in the projectile frame is a Maxwellian distribution with some temperature $T$, then for an integral frequency distribution the logarithm of $F(>Q)$ is

$$
\operatorname{InF}(>Q) \approx-\left(A / 2 / M_{p}\right) \cdot(q / T)
$$

Linearity of such a plot is a strong evidence for a single temperature $T$ describing this type of interaction. The authors of [4] continued: "We selected events with $n_{\alpha} \geq 2$ [ $n_{\alpha}$ being the number of alphas in one specific event] in which at least one He fragment had a transverse momentum exceeding some threshold value $Q_{t h}$,

$Q_{\mathrm{th}}=Q_{0}=0.02[(\mathrm{GeV} / \mathrm{c}) / \text { nucleon }]^{2}$, chosen so that it corresponds to practical extinction of a $T=10 \mathrm{MeV}$ component. The trigger particle with the highest $q$ [transverse momentum of trigger particle] was excluded from the analysis and the remaining alpha particle momenta were plotted. In the same way we proceeded with those events in which no $\alpha$ particles occurred with a momentum $q>Q_{0}$.

The results are shown in Figure 2. The surprising finding is that the residual particles from the tagged events appear to constitute a homogeneous group corresponding to a temperature of $T(p)_{\text {hot }}=40 \mathrm{MeV}$ (hot events; Figure 2(a)). When events in which the highest $q$ of a He particle lies below $Q_{0}$ are plotted in the same way one finds a quite different but again homogeneous distribution corresponding to a much lower $T(p)_{\text {cool }} \approx 10 \mathrm{MeV}$, which is approximately the boiling temperature of the nucleus (cool events; Figure 2(b)).

One observes about $80 \%$ of all events in the "cool group" and about $20 \%$ in the "hot group".

Ganssauge et al. [7,8] reproduced the essential results of Baumgardt et al. [4] with an independent study of nu- 


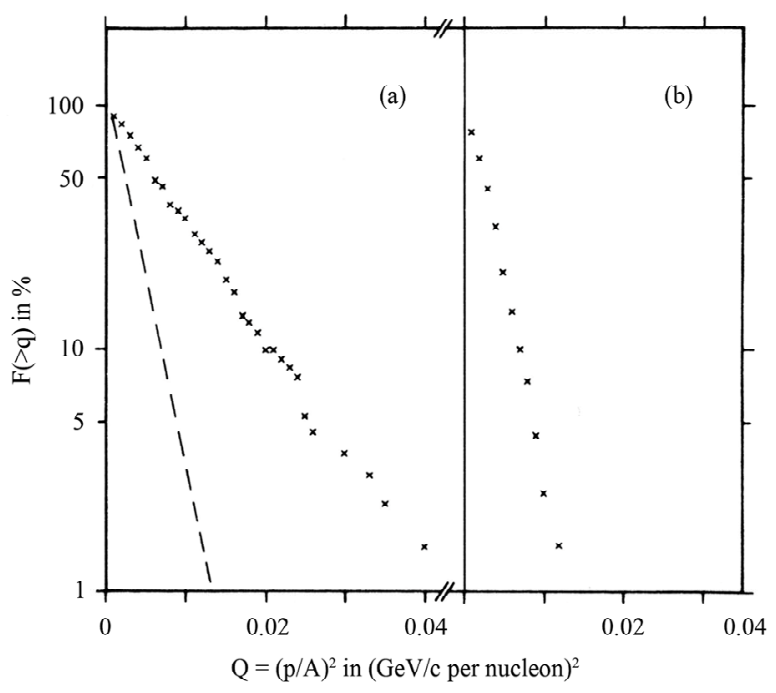

Figure 2. Normalized integral frequency distribution $F(>q)$ for He projectile fragments, omitting the trigger particles: (a) $q_{\text {trigger }}>Q_{0}=0.02[(\mathrm{GeV} / \mathrm{c}) / \text { nucleon }]^{2}$, (hot events, $T(p)_{\text {hot }}$ $\approx 40 \mathrm{MeV}, \sim 20 \%$ ). (b) $q_{\text {trigger }} \leq Q_{0}$ (cool events, $T(p)_{\text {cool }} \approx 10$ $\mathrm{MeV}, \sim 80 \%$ ). The dashed line in (a) depicts the distribution from (b).

clear emulsions irradiated with $100 \mathrm{GeV}{ }^{56} \mathrm{Fe}$ ions. Joseph et al. [9] did the same with nuclear emulsions irradiated with $72 \mathrm{GeV}^{40} \mathrm{Ar}$ ions, and Aggarwal et al. [10] studied also the same interactions as the two preceding groups. All groups started their investigations with new irradiations of nuclear emulsion. The number of analyzed nuclear events was always substantially larger in [7-10] than in [4]. All groups confirmed the central observation of Baumgardt et al.:

\section{There Are Two Independent Kinds of Interactions Observed for Projectile-Like Fragments}

In about $80 \%$ of all events one observes nothing exciting, these are "cool" interactions. In about $20 \%$ of all interactions one observes a "hot" source emitting projectile fragments. Both channels are clearly separated.

As an example of the other independent measurements, the normalized frequency distribution for He projectile fragments showing two independent reaction channels is depicted in Figure 3. The data are taken from [8] where the authors followed the procedure of [4] in their separation of "cool" from "hot" events. Of particular interest is the indication of a third and even "hotter" temperature component within the "hot" regime $(<10 \%)$. Actually, all three groups observed this phenomenon within the "hot" distribution, but omitted a more detailed and quantitative analysis [7-10].

Joseph et al. [9] summarized their results: "This high statistics study based on the analysis of 3308 relativistic alphas gives clear evidence of two distinctly different

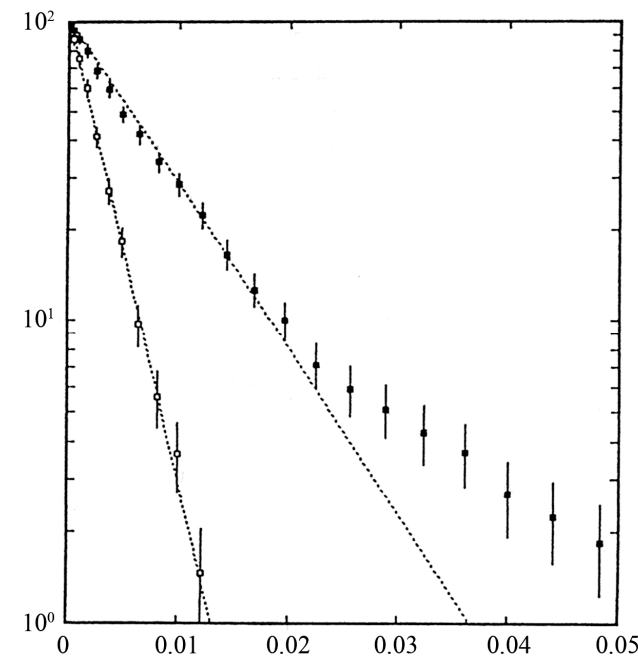

Figure 3. Normalized integral frequency distribution $F$ $\left(>\left\{\left[p_{t} / A^{2}\right][\mathrm{GeV} / \mathrm{c}]^{2}\right\}\right)$ for residual He projectile fragments, omitting the trigger particles: right, hot events $\left(T(p)_{\text {hot }} \approx 43\right.$ $\mathrm{MeV}, \sim 20 \%)$; left, cool events $\left(T(p)_{\text {cool }} \approx 10 \mathrm{MeV}, \sim 80 \%\right)$ [8].

temperatures in the fragmentation region." Aggarwal et al. [10] reached similar conclusions.

\section{In Primary Interactions ONLY ONE TYPE of Target-Like Fragments Is Produced}

The complementary partners to projectile-like fragments in these interactions are the target-like fragments appearing as heavy tracks. They are isotropically emitted in the lab system (see Figure 1). $\mathrm{T} \mathrm{N}_{\mathrm{h}}^{2}$ he authors of [4] discussed this only shortly and did not publish all relevant details. In the context of questions discussed in this paper, there exists only one experiment - the irradiation of nuclear emulsions with $100 \mathrm{GeV}^{56} \mathrm{Fe}$ - where the number of heavy tracks, $\mathrm{N}_{\mathrm{h}}$, coming from target-like fragments has been studied systematically. Ganssauge and his group investigated target-like fragments for "cool" and "hot" events $[7,8]$ and presented a normalized frequency distribution of the target-like fragment multiplicity $\mathrm{N}_{\mathrm{h}}$ for "cool" and "hot" reaction channels (Figure 4).

The average $\left\langle\mathrm{N}_{\mathrm{h}}\right\rangle$ is for "cool" events: $\left(\left\langle\mathrm{N}_{\mathrm{h}}\right\rangle\right)_{\text {cool }}=$ $(9.1 \pm 0.5)$ tracks/event, and for "hot" events: $\left(\left\langle\mathrm{N}_{\mathrm{h}}\right\rangle\right)_{\text {hot }}$ $=(11.4 \pm 1.4)$ tracks/event.

A more detailed analysis shows that "hot" events are found slightly more often due to interactions with Ag- or Br-target nuclei as compared to "cool" interactions, as it was observed already in [4]. Only on heavy target nuclei (Ag and $\mathrm{Br}$ ) one can find reactions with more than 8 tracks $\left(\mathrm{N}_{\mathrm{h}} \geq 9\right)$. Table 1 gives a summary of relevant experimental results for $\mathrm{N}_{\mathrm{h}}$-distributions from [8].

According to semi-empirical standard procedures in nuclear emulsion experiments, such as described in the 
Table 1. Number of black prongs, $N_{h}$, in a nuclear emulsion irradiated with $100 \mathrm{GeV}^{56} \mathrm{Fe}$.

\begin{tabular}{|c|c|c|c|c|}
\hline & $\mathrm{N}_{\mathrm{h}} \leq 8$ & $\mathrm{~N}_{\mathrm{h}} \geq 9$ & $\mathrm{~N}_{\mathrm{h}} \geq 0$ & $\left(N_{h} \geq 9\right) /\left(N_{h} \leq 8\right)$ \\
\hline \multirow[t]{2}{*}{ "cool", $n_{\alpha} \geq 2$} & 222 events $(62 \%)$ & 136 events (38\%) & 358 events $(100 \%)$ & $0.61 \pm 0.07$ \\
\hline & $<\mathrm{N}_{\mathrm{h}}>=3.0 \pm 0.3$ & $<\mathrm{N}_{\mathrm{h}}>=19.0 \pm 1.7$ & $<\mathrm{N}_{\mathrm{h}}>=9.1 \pm 0.5$ & $6.3 \pm 0.5$ \\
\hline \multirow[t]{2}{*}{ "hot", $n_{\alpha} \geq 2$} & 38 events $(49 \%)$ & 40 events $(51 \%)$ & 78 events $(100 \%)$ & $1.05 \pm 0.22$ \\
\hline & $<\mathrm{N}_{\mathrm{h}}>=3.3 \pm 0.6$ & $<\mathrm{N}_{\mathrm{h}}>=19.1 \pm 3.1$ & $<\mathrm{N}_{\mathrm{h}}>=11.4 \pm 1.3$ & $5.8 \pm 1.0$ \\
\hline
\end{tabular}

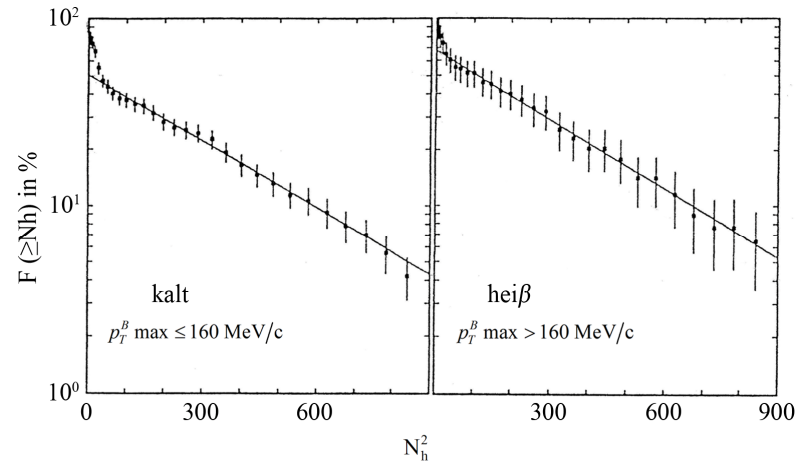

Figure 4. Normalized integral frequency distribution $F\left(>N_{h}\right)$ for target-like fragments in the "cool" (kalt) and "hot" (heiß) reaction channels $[8]$.

textbook of Powell et al. [6], one can correlate the average number of heavy tracks $<\mathrm{N}_{\mathrm{h}}>$ with a nuclear heat $\mathrm{U}$ as follows:

$$
\mathrm{U}\left(\left\langle\mathrm{N}_{\mathrm{h}}\right\rangle\right)=\left(124\left\langle\mathrm{~N}_{\mathrm{h}}\right\rangle+30\right) \mathrm{MeV}
$$

and $\mathrm{U}$ is connected with the nuclear temperature $T$ as:

$$
T=\left\{(10 / A) \cdot \mathrm{U}\left(\left\langle\mathrm{N}_{\mathrm{h}}\right\rangle\right)\right\}^{1 / 2} \mathrm{MeV}
$$

Combining Equations 4 with 5 yields:

$$
T=\left\{(10 / A) \cdot\left(124\left\langle\mathrm{~N}_{\mathrm{h}}\right\rangle+30\right)\right\}^{1 / 2} \mathrm{MeV}
$$

Using these formulas, one obtains for the "cool" and "hot" target-like fragments about the same nuclear temperature:

$$
T\left(\left\langle\mathrm{~N}_{\mathrm{h}}\right\rangle\right)_{\text {cool }} \approx T\left(\left\langle\mathrm{~N}_{\mathrm{h}}\right\rangle\right)_{\mathrm{hot}} \approx 12 \mathrm{MeV}
$$

This temperature of $12 \mathrm{MeV}$ is based on the assumption of an average mass $A=80$ of the target atoms within the nuclear emulsion. Therefore this temperature is only a rough estimate. However, the essential result of equation 7 remains valid: The nuclear temperatures in both channels, the "cool" and "hot" target-like sources, must be quite similar. Actually, this is the temperature of a "cool source".

Experimental results of derived temperatures vs. $\left\langle\mathrm{N}_{\mathrm{h}}\right\rangle$ are shown in Figure 5.

It is of interest to note, that the behavior of $\left\langle\mathrm{N}_{h}\right\rangle$ as observed in this paper, has been known for a long time. As early as 1967, Friedlander and Friedman [11] measured a

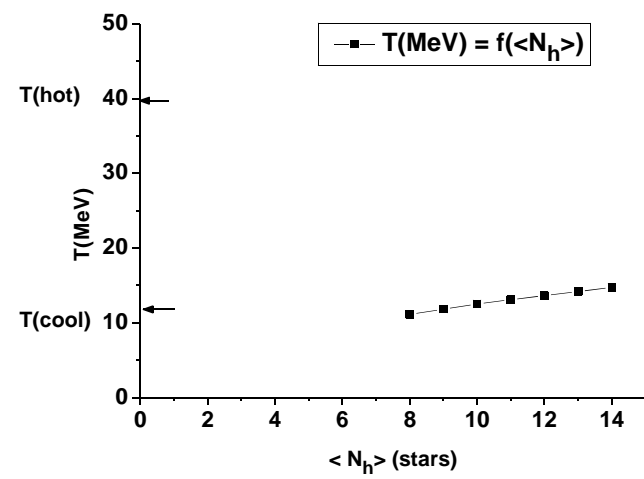

Figure 5. Increase of the nuclear temperature $T$ with the number $\left\langle\mathrm{N}_{\mathrm{h}}\right\rangle$ of heavy prongs according to equation 6 . Taking the experimental values for $\left\langle\mathrm{N}_{\mathrm{h}}>\right.$ from Table 1 , one obtains only a "cool" temperature of $T(\mathrm{cool}) \approx 12 \mathrm{MeV}$, but there is NO WAY to reach a source temperature of $T($ hot $) \approx$ $40 \mathrm{MeV}$.

similar integral frequency distribution $F\left(>\mathrm{N}_{\mathrm{h}}\right)$ in interacttions induced by cosmic rays in nuclear emulsions. The primary energy of cosmic rays observed in these nuclear emulsions extended up to $2000 \mathrm{GeV}$.

Not surprisingly, Friedlander and Friedmann nevertheless observed only well-known "cool" phenomena in their $\left\langle\mathrm{N}_{\mathrm{h}}\right\rangle$ distributions in [11], as also reported in [4]. The authors of [11] simply did not study projectile-like fragments so they could not see the hot regime.

It was shown in [1] that the threshold of $E_{\mathrm{cm}} / u \approx 150$ $\mathrm{MeV}$ applies for all hadronic (protons and heavier ions) primary interactions. All cosmic rays with energy above about $30 \mathrm{GeV}$ should therefore induce "unresolved problems" in emulsion.

\section{4. “Hot" Relativistic Projectile Fragments Are an Unresolved Problem}

The essential results of this article shall be repeated:

For projectile-like fragments one observes two kinds of reactions with distinctly different temperatures $T(p)$ in the "cool" and "hot" reaction channels:

$T(p)_{\text {cool }} \approx 10 \mathrm{MeV}, T(p)_{\text {hot }} \approx 40 \mathrm{MeV}$.

For target-like fragments one observes from both reaction channels:

$$
T\left(\left\langle\mathrm{~N}_{\mathrm{h}}\right\rangle\right)_{\text {cool }} \approx T\left(\left\langle\mathrm{~N}_{\mathrm{h}}\right\rangle\right)_{\text {hot }} \approx 12 \mathrm{MeV} .
$$

This has been considered an unresolved problem al- 
ready in 1987 [8]:

"The strong increase of projectile-like temperatures from 'cool' events to 'hot' events has no correspondence on the target-like side".

The unresolved problem shows up in primary ion interactions above the threshold given earlier $\left(E_{\mathrm{CM}} / u \approx 150\right.$ $\mathrm{MeV}$ ) — as far as the limited experimental evidence allows definition. The authors of this paper have no problem to understand the "cool" reaction path, as these classical spallation reactions have been studied for decades using well-known concepts of nuclear science [3]. However, we are not aware of any model calculation, which yields such a strong difference in ONE nuclear interaction leading to rather conventional temperatures in the target-like residuals and simultaneously to exceedingly high temperatures for $20 \%$ of the projectile-like fragments. There appears to be a problem with classical concepts, which always request an increase in entropy for reactions in isolated systems. One should remember: the volume for this interaction has only a tiny radius (approx. $10^{-12} \mathrm{~cm}$ ) and the reaction time is very short (approx. $10^{-20} \mathrm{~s}$ ).

In this situation it might be allowed to recall a historical paper of L. Szilard, published in Berlin in 1929: "Über die Entropieverminderung in einem thermodynamischen System bei Eingriffen intelligenter Wesen." [12].

Instead of trying a translation, we employ a description of the essence of this article as given by Szilard's friend E. Teller in his Memoirs [13]:

"Szilard proposed that the rules of thermodynamics remain valid, if one includes the demon in the system rather than talking exclusively about the state of disorder (entropy). He assigned a value of entropy (or of order) to pieces of information received by the demon. Szilard's work gave rise to a practical and famous branch of science, called information theory, important today in transmitting information at minimum cost."

This ansatz with an active demon interfering with a nuclear reaction system may turn out be useful or not-it should at least be mentioned. Obviously, information theory and physics of high energy nuclear interactions are quite different fields. In this nuclear application, the demon will not receive information, but the demon may introduce information into a nuclear system and thus create a new experimental situation, i.e. the separation of "hot" projectile-like fragments from "cool" target-like residuals. (One may ask: is the demon introducing some "Ordnung"?). One might investigate if the ideas of Szilard could be fruitful even in this new field.

Future experiments with heavy ion induced reactions above the threshold of $\left(E_{\mathrm{CM}} / u \approx 150 \mathrm{MeV}\right)$ will answer the questions: Is the "hot" channel in the first interaction responsible for producing unresolved problems in consecutive second interactions? So far, this problem has not been settled in a definite and conclusive experiment. Do "hot" projectile-like He-fragments produced in $100 \mathrm{GeV}$

${ }^{56} \mathrm{Fe}$ interactions show in their $2^{\text {nd }}$ interactions within the same nuclear emulsion any specific particular characteristics, for example enhanced $\mathrm{N}_{\mathrm{h}}$ yields? Such studies can also be done in nuclear emulsions irradiated with $72 \mathrm{GeV}$ ${ }^{40} \mathrm{Ar}$ ions. Preliminary studies of the $2^{\text {nd }}$ interactions in nuclear emulsions irradiated with $72 \mathrm{GeV}^{22} \mathrm{Ne}$ have been mentioned in [3]. Their results could not answer the questions at stake in a conclusive manner. However, this last study did not consider specifically He-like "hot" projectile fragments.

Are "hot" He-like projectile fragments responsible for secondary interactions inducing the enhanced neutron emission and enhanced nuclear destruction reported earlier in [1-3]? This problem cannot be studied directly with radiochemical methods or neutron yield measurements, but results from these two independent experimental techniques would be useful to obtain a complete picture of this new nuclear process. In a recent conference contribution [14] "Suggested investigations to understand experimental observables" more experimental studies were proposed. Among others, these experiments are geared towards answering the question of the origin of various unexplained experimental results, such as e.g.:

1) The extremely large neutron fluence at a distance of over 240 meters from the $20 \mathrm{~cm}$ THICK Cu target irradiated with $72 \mathrm{GeV}^{40} \mathrm{Ar}$ ions at the BEVALAC in Berkeley (USA) in March 1987 (see Appendix), or the 3-fold increase of the neutron fluence generated with $44 \mathrm{GeV}{ }^{12} \mathrm{C}$ onto $20 \mathrm{~cm} \mathrm{Cu}$ (or $\mathrm{Pb}$ ) at the JINR accelerator in Dubna (Russia) as compared to most recent calculations.

2) Radiochemical spallation yields in THICK $\mathrm{Cu}$ targets irradiated with the same ions as above show an enhanced nuclear destruction beyond standard model expectations.

These questions are directly connected with the design of radiation protection shielding in high energy heavy ion accelerators presently under construction.

\section{REFERENCES}

[1] W. Westmeier, R. Brandt, S. R. Hashemi-Nezhad, R. Odoj, A. N. Sosnin, W. Ensinger and M. Zamani-Valasiadou, "Correlations in Nuclear Interactions between $\mathrm{E}_{\mathrm{CM}} / \mathrm{u}$ and Unexplained Experimental Observables," World Journal of Nuclear Science and Technology, Vol. 2, 2012, pp. 125-132. http://dx.doi.org/10.4236/wjnst.2012.24018

[2] S. R. Hashemi-Nezhad, M. Zamani-Valasiadou, M. I. Krivopustov, R. Brandt, W. Ensinger, R. Odoj and W. Westmeier, "Neutron Production in Thick Targets Irradiated with High Energy Ions," Physics Research International, Vol. 2011, 2011, Article ID: 128429.

[3] R. Brandt, V. A. Ditlov, K. K. Dwivedi, W. Ensinger, E. Ganssauge, Guo Shi-Lun, M. Haiduc, S. R. Hashemi-Nezhad, H. A. Khan, M. I. Krivopustov, R. Odoj, E. A. Po- 
zharova, V. A. Smirnitzki, A. N. Sosnin, W. Westmeier and M. Zamani-Valasiadou, "Interactions of Relativistic Heavy Ions in Thick Heavy Element Targets and Some Unresolved Problems," Physics of Particles and Nuclei, Vol. 39, No. 2, 2008, pp. 259-285.

[4] H. G. Baumgardt, E. M. Friedlander and E. Schopper, "Evidence for Two Different Reaction Mechanism in Relativistic Heavy-Ions Collisions," Journal of Physics G: Nuclear and Particle Physics, Vol. 7, 1981, pp. L175L181. http://dx.doi.org/10.1088/0305-4616/7/8/004

[5] E. Ganssauge, "Anomalons," Proceedings of International School of Physics "Enrico Fermi," Nuclear Structure and Heavy-Ion Dynamics, North-Holland Publications, 1984, pp. 551-582.

[6] C. F. Powell, P. H. Fowler and D. H. Perkins, "The Study of Elementary Particles by the Photographic Method. An Account of the Principal Techniques and Discoveries Illustrated by an Atlas of Photomicrographs," Pergamon Press Ltd., London, New York, 1959.

[7] E. Ganssauge, H. Kallies, B. Dressel, Ch. Müller and W. Schulz, "Two Distinct Classes of Alpha Particles and a Possible Correlation of Anomalously Short Mean Free Path with the Cold Component," Journal of Physics G: Nuclear and Particle Physics, Vol. 11, 1985, pp. L139L142. http://dx.doi.org/10.1088/0305-4616/11/8/004

[8] H. Kallies, "Zum Verhalten von Projektilfragmenten der Ladung $Z=2$ bei Relativistischen Kern-Kern-Stössen in Kernspuremulsionen," PhD Thesis, Fachbereich Physik, Philipps-Universität, Marburg, 1987 (unpublished).
[9] R. R. Joseph, I. D. Ojha, S. K. Tulit, V. S. Bhatia, M. Kaur, I. S. Mittra, S. S. Sahota, K. B. Bhalla, A. Bharti, S. Mookerjee, S. Kitroo and N. K. Rao, "Two Source Emission of Relativistic Alpha-Particles in ${ }^{40}$ Ar-Emulsion Collisions," Journal of Physics G: Nuclear and Particle Physics, Vol. 15, 1989, pp. 1805-1814. http://dx.doi.org/10.1088/0954-3899/15/12/007

[10] M. M. Aggarwal, K. B. Bhalla, G. Das and P. L. Jain, “Angular Distributions of Relativistic Alpha Particles in Heavy-Ion Collisions," Physical Review C, Vol. 27, 1983, pp. 640-649. http://dx.doi.org/10.1103/PhysRevC.27.640

[11] E. M. Friedlander and A. Friedmann, "Frequency Distributions of Heavy Prongs from High-Energy Stars in Nuclear Emulsions," Nuovo Cimento A, Vol. 52, 1967, pp. 912-917. http://dx.doi.org/10.1007/BF02738852

[12] L. Szilard, "Über die Entropieverminderung in Einem Thermodynamischen System bei Eingriffen Intelligenter Wesen," Zeitschrift für Physik, Vol. 53, 1929, pp. 840866. http://dx.doi.org/10.1007/BF01341281

[13] E. Teller, "Memoires (a Twentieth Century Journey in Science and Politics)," Perseus Publishing, 2001, Lib. of Congress, p. 111.

[14] W. Westmeier, R. Brandt and S. Tyutyunnikov, "Suggested Investigations Concerning Unresolved Experimental Observations," XXI International Baldin Seminar on High Energy Physics Problems, 10-15 September 2012, Dubna. 


\section{Appendix}

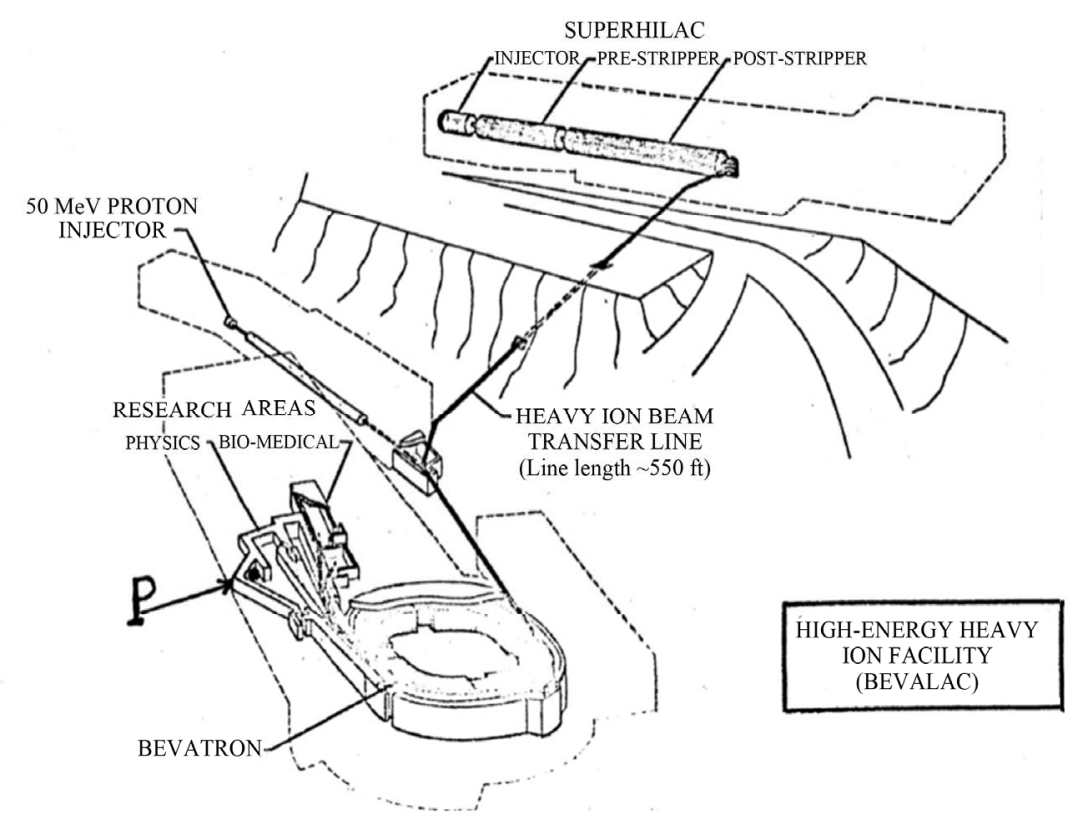

Figure A1. Sketch of parts of the Lawrence Berkeley National Laboratory (California). with its former BEVALAC (HighEnergy Heavy Ion Accelerator) and the position "P", where a $20 \mathrm{~cm}$ thick copper target was irradiated for 44 minutes with $72 \mathrm{GeV}^{40} \mathrm{Ar}$-ions ( $\sim 5 \times 10^{8}$ ions/second) on March 10, 1987. The health physics neutron monitoring measurements at the SUPERHILAC, at a distance of about $240 \mathrm{~m}$ from " $P$ ", forced the operators to announce: "There is a large neutron dose above the warning threshold within the entire laboratory". No further information about the actually measured neutron dose was ever released. 